\title{
Evaluation of S1PR1, pSTAT3, S1PR2, and FOXP1 expression in aggressive, mature $B$ cell lymphomas
}

\author{
Mustafa Al-Kawaaz ${ }^{1} \cdot$ Teresa Sanchez $^{1,2} \cdot$ Michael J. Kluk $^{1}$ \\ Received: 28 December 2018 / Accepted: 15 April 2019 / Published online: 27 April 2019 \\ (C) Springer-Verlag GmbH Germany, part of Springer Nature 2019
}

\begin{abstract}
Aggressive, mature B cell lymphomas include Burkitt lymphoma (BL); high-grade B cell lymphomas (HGBL) (e.g., double-hit B cell lymphomas (HGBL-DH: HGBL with MYC and BCL2 and/or BCL6 translocations)); HGBL, not otherwise specified (HGBL, NOS); and diffuse large B cell lymphoma (DLBCL). Overlapping morphologic and immunohistochemical features of these lymphomas pose diagnostic challenges in some cases, and better understanding of potential diagnostic biomarkers and possible therapeutic targets is needed. Sphingosine 1 phosphate receptors (S1PR1-5) are G protein-coupled receptors that bind S1P and influence migration and survival in multiple cell types, including lymphocytes. S1PRs are emerging as biomarkers in B cell biology and interaction between S1PR pathways and STAT3 or FOXP1 has been reported in DLBCL. Our aim was to extend the understanding of S1PR1, STAT3, and S1PR2, FOXP1 expression beyond DLBCL, into additional aggressive, mature B cell lymphomas using immunohistochemical expression analysis of human tissue samples. S1PR1 and S1PR2 showed different expression patterns in mantle zones and follicle centers in reactive lymphoid tissue. BL showed a unique expression pattern compared to HGBL and DLBCL. Additionally, S1PR1 and S1PR2 expression were typically mutually exclusive and were expressed in a low proportion of cases (frequently HGBL involving extranodal sites). FOXP1 was expressed in a high proportion of various case types and pSTAT3 was detected in a significant proportion of HGBL and DLBCL. These findings provide further evidence that S1PR1, pSTAT3, S1PR2, and FOXP1 play a role in a subset of aggressive, mature B cell lymphomas.
\end{abstract}

Keywords Sphingosine-1-phosphate $\cdot \mathrm{S} 1 \mathrm{P} \cdot \mathrm{S} 1 \mathrm{PR} \cdot \mathrm{Lymphoma} \cdot \mathrm{pSTAT3} \cdot \mathrm{FOXP1}$

\section{Introduction}

Aggressive, mature B cell lymphomas represent a heterogeneous group of diseases including Burkitt lymphoma (BL); high-grade B cell lymphomas (HGBL) (e.g., Double-Hit B cell lymphomas (HGBL-DH: HGBL with MYC and BCL2 and/or BCL6 translocations)); HGBL, not otherwise specified (HGBL, NOS)); and diffuse large B cell lymphoma (DLBCL). DLBCL can be further classified into germinal center B celllike (GCB subtype) and activated B cell-like (ABC; non-GCB subtype) $[1,2]$. In some cases, classification of these types of

Michael J. Kluk

mik9095@med.cornell.edu

1 Department of Pathology and Laboratory Medicine, Weill Cornell Medicine, 1300 York Ave. Office: K509, New York, NY 10065, USA

2 Department of Neuroscience, Brain and Mind Research Institute, Weill Cornell Medicine, New York, NY, USA aggressive mature B cell lymphomas can pose diagnostic challenges, and due to their aggressive clinical course, a better understanding of diagnostic biomarkers and potential therapeutic targets is needed.

Sphingosine 1 phosphate receptors (S1PR1-5) represent a family of $G$ protein-coupled receptors that bind the sphingolipid (S1P) and influence migration and survival pathways in a variety of cell types; S1PRs are emerging as biomarkers in B cell lymphomas [3-6] and B cell development [7]. Our prior work, which demonstrated S1PR1 expression in a subset of classic Hodgkin lymphoma cases [8], has recently been supported by others [9]. Additional studies indicate that S1PR expression may influence anatomic location/ distribution in a variety of types of lymphoma [10]. Although the role of S1PR1 and S1PR2 have been examined in mantle cell lymphoma and DLBCL, the role of this S1P pathway in BL and other HGBL, including HGBL-DH has not been specifically characterized.

STAT3 is a transcription factor which regulates tumorigenesis in a variety of lymphoproliferative disorders and is 
therapeutically targetable $[11,12]$. pSTAT3 has been reported to be a potential biomarker in DLBCL which may depend on anatomic location, according to one study [13]. STAT3 was reported to show high levels of expression and phosphorylation in ABC-DLBCL [14]. Interestingly, STAT3 was found to be a direct transcriptional activator of S1PR1 expression in various cell types [15]. In DLBCL, S1PR1 over-expression has been reported in approximately $6-40 \%$ of DLBCL and was associated with STAT3 phosphorylation in fresh tissue and as well as a negative prognosis [5, 16]. Additional studies, using primary tumor cells, have also shown phospho-STAT3 activity correlated with increased S1PR1 expression in ABCDLBCL [17], and that S1PR1 could activate STAT3 in ABCDLBCL [17]. Furthermore, inhibition of S1PR1 expression, downregulated STAT3 activity and caused growth inhibition of lymphoma cells [17]. In BL, phosphorylated STAT3 was reported to be associated with multidrug resistance according to one study [18]; however, the expression of phosphorylated STAT3 does not appear to have been adequately examined using clinical material. In terms of other HGBL, phosphorylated STAT3 has been reported to be associated with MYC and MYC/BCL2 double expression in one study [19]; however, there was no association between phosphorylation of STAT3 with rearrangement of MYC, BCL2, or BCL6.

FOXP1 (Forkhead box protein $\mathrm{P} 1$ ) is a transcription factor that is expressed in a subset of GCB-DLBCL, and to a greater extent in ABC-like DLBCL, and may be associated with a poorer overall survival according to some studies $[13,20]$. According to some studies, FOXP1 expression correlates with MYC expression [20] and in a limited series of 11 cases of triple-hit lymphoma (i.e., B cell lymphomas with MYC, BCL2, and BCL6 translocations), all 11 cases were positive for FOXP1 [21]. However, FOXP1 expression in BL and HGBL-DH has yet to be adequately explored. Furthermore, interestingly, FOXP1 was reported to repress S1PR2 expression in ABCand GCB-DLBCL cell lines and FOXP1 mRNA expression was inversely correlated with S1PR2 mRNA expression in patient cohorts; additionally, low S1PR2 mRNA expression, especially together with high FOXP1 mRNA expression, was associated with a negative prognosis [22]. In additional studies, S1PR2 was found to play a role in germinal center confinement of B cells [23] and mutations in S1PR2 reported in GCB-DLBCLs were found to disrupt the inhibitory functions of this receptor [6, 24, 25].

Taken together, the prior research suggests a role for S1PR1, pSTAT3, S1PR2, and FOXP1 in distinct subtypes of DLBCL; however, testing for these biomarkers in additional patient cohorts and disease subsets is needed. Therefore, our aim was to extend the understanding of S1PR1, pSTAT3, S1PR2, and FOXP1 expression beyond DLBCL into additional aggressive, mature $\mathrm{B}$ cell lymphomas such as $\mathrm{BL}$; HGBL-DH; and HGBL, NOS.

\section{Materials and methods}

Formalin-fixed paraffin embedded (FFPE) tissue samples from 150 total cases were identified from the Weill Cornell Medicine/New York Presbyterian Hospital, Department of Pathology archives. The cases were diagnosed by subspecialist hematopathologists; $98 \%$ (42/43 cases) of HGBL, BL, and BLL cases were originally diagnosed after 2008 per the WHO 2008 guidelines (4th edition) [1]. Ninety-one percent (97/107) of DLBCL cases were originally diagnosed after 2008 per the WHO 2008 guidelines (4th edition). During the collection of the original diagnoses for this study, the latest WHO nomenclature (revised 4th edition, 2017) [2] was used when cytogenetic data was available which would permit using the updated nomenclature (e.g., HGBCL, DH, and the case of Burkitt-like lymphoma with 11q aberration). The original cytogenetic/fluorescent in situ hybridization (FISH) data was used for this study and was available in $96 \%$ (27/28 cases) of HGBL and BLL cases and in 63\% (67/107) of DLBCL cases. Immunohistochemistry (IHC) for S1PR1, pSTAT3, S1PR2, and FOXP1 was performed on the Leica Bond Autostainer (Buffalo Grove, IL.) on paraffin embedded tissue sections. We have previously validated the S1PR1 antibody [8] (Santa Cruz, SC-25489, H60); the S1PR1 staining conditions in this study were H2(tris-EDTA(pH 9)) antigen retrieval for $20 \mathrm{~min}$ and 30 min incubation with primary antibody (dilution of 1:100). S1PR2 (Acris, AP0-1198PU-N) performance was confirmed (Fig. 1) and staining conditions were H2(Tris$\operatorname{EDTA}(\mathrm{pH}$ 9)) antigen retrieval for $20 \mathrm{~min}$ and 15 min incubation with primary antibody (dilution of 1:100). Cases were positive for S1PR1 or S1PR2 when $\geq 50 \%$ of lymphoma cells showed moderate to strong cytoplasmic and/or membranous staining; this S1PR1 cutoff was set to be slightly higher than that reported previously $[5,16]$, so that the positive cases comprise samples in which a significant portion of tumor cells were S1PR1 positive. A precedence for a cutoff for S1PR2 positivity in tumor cells upon IHC staining of human tissues is not established in the literature. FOXP1 (ABCAM, AB16645) staining conditions were $\mathrm{H1}(\mathrm{Citrate}(\mathrm{pH} 6))$ antigen retrieval for $30 \mathrm{~min}$ and $15 \mathrm{~min}$ incubation with primary antibody (dilution 1:400). Cases were positive for FOXP1 when $>90 \%$ ( $2 / 2$ level positivity) or $50-90 \%$ ( $1 / 2$ level positivity) of lymphoma cells were positive for nuclear staining. These FOXP1 cutoffs are similar to various previously published FOXP1 cutoffs [20]. Phospho-STAT3 (cell signaling, CST 9145; Tyr705) staining conditions were H2(Tris-EDTA(pH 9)) antigen retrieval for $20 \mathrm{~min}, 60 \mathrm{~min}$ incubation with primary antibody(dilution 1:100). Cases were positive for phosphoSTAT3 when $>10 \%$ of lymphoma cells showed nuclear staining, since phosphoepitopes are a class of immunostaining markers that may not be as well preserved as other antigens in formalin fixed tissues; other studies have also used low, variable cutoffs for p-STAT3 positivity [26, 27]. Phospho- 
Fig. 1 a-d Immunostaining for S1PR1 (a), S1PR2 (b), FOXP1 (c), and pSTAT3 (d) in follicles from reactive lymphoid tissue; S1PR1 staining is seen mainly in mantle zones and vascular endothelial cells rather than reactive follicle centers; S1PR2 staining is mainly seen in reactive follicle centers and vascular endothelium, rather than mantle zones; FOXP1 is most highly expressed in mantle zones; pSTAT3 stains scattered cells, including endothelial cells and stromal cells $(\times 10(\mathbf{a}, \mathbf{b})$ or $\times 20$ (c, d) magnification; scale bars $=$ $200 \mu \mathrm{m}(\mathbf{a}, \mathbf{b})$ or $100 \mu \mathrm{m}(\mathbf{c}, \mathbf{d}))$. e-g Immunostaining for S1PR2 is shown for S1PR1-transfected (e), S1PR2-transfected (f), and control vector-transfected (g) human embryonic kidney 293 cells; S1PR2 staining is appropriately seen for S1PR2-transfected cells, but not for S1PR1- or control vector-transfected cells $(\times 40$ magnification, scale bars $=$ $50 \mu \mathrm{m})$
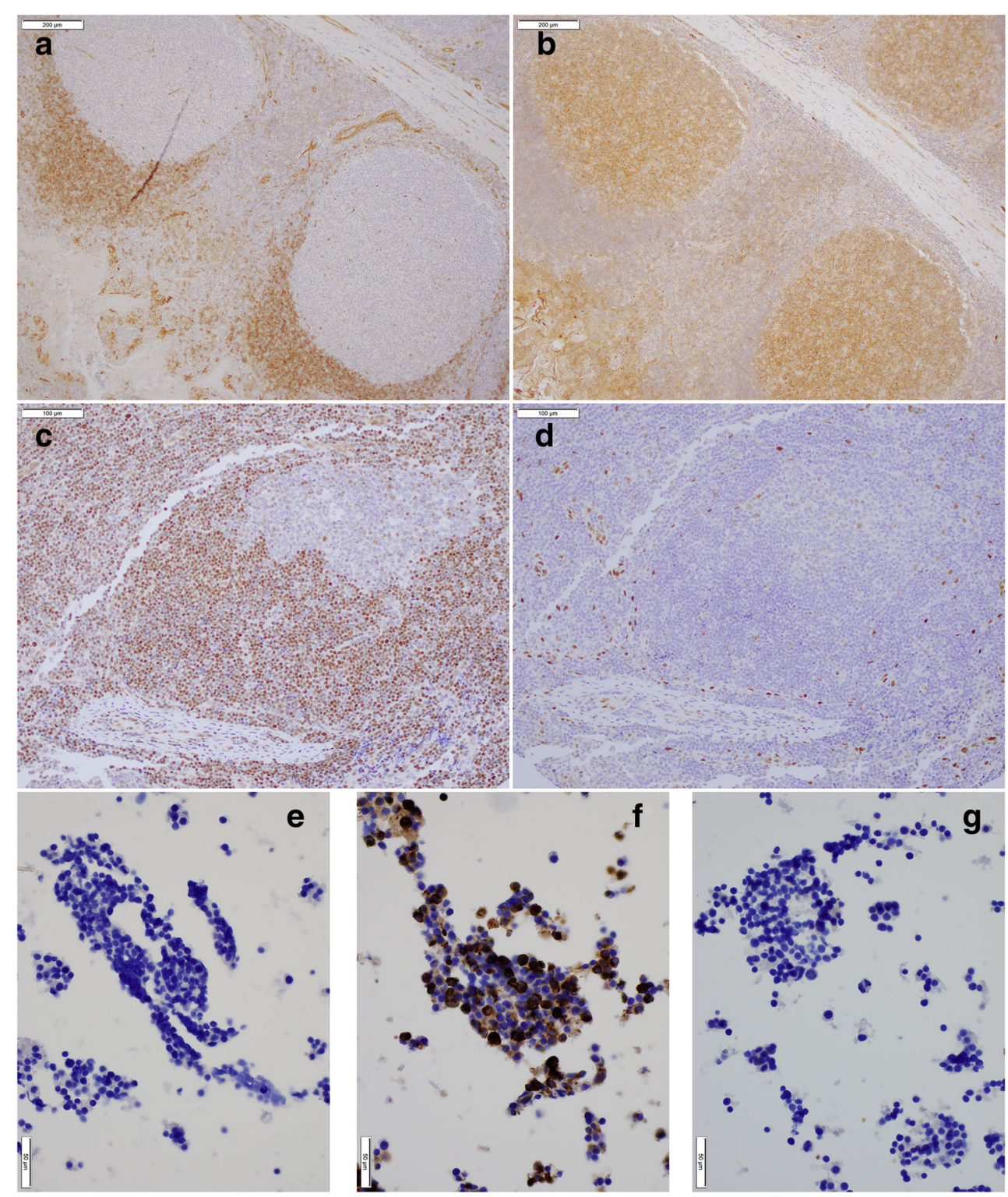

STAT3 showed nuclear positivity in vascular endothelial cells in control tissues as previously reported $[5,16]$. Vascular endothelial cell and/or stromal cell staining (internal control) was present for each marker in cases when lymphoma cells were negative. This work was supported by the Translational Research Program at WCMC Pathology and Laboratory Medicine and was approved by the Weill Cornell Institutional Review Board (Protocol \#: 1509016528).

\section{Results}

Immunostaining for S1PR1 showed findings consistent with the reported staining pattern [4], whereby mantle zones and vascular endothelial cells were S1PR1 positive, while follicle centers were negative for S1PR1 (Fig. 1). On the other hand,
S1PR2 was expressed more strongly in follicle centers than in mantle zones (Fig. 1), which is consistent with the S1PR2 mRNA expression studies described previously in mice [23] and sorted human tonsillar B cells [24]; in addition, S1PR2staining was specific for S1PR2 when tested with control cell lines (Fig. 1). FOXP1 staining was consistent with previously described patterns [20], such that nuclear FOXP1 was more strongly expressed in mantle zone lymphocytes than follicle center lymphocytes (Fig. 1). Lastly, phospho-STAT3 showed scattered staining in reactive follicles and interfollicular areas, including vascular endothelial cell nuclei and scattered stromal cell nuclei (Fig. 1), consistent with reported staining patterns $[5,16]$.

A total of 150 cases of aggressive B cell lymphoma were tested. The staining results are summarized in Table 1 and representative images are shown (Fig. 2). The cases comprised 
Table 1 Summary of immunostaining results

\begin{tabular}{|c|c|c|c|c|c|}
\hline Diagnosis & Number of cases & S1PR1 positive, $n(\%)$ & pSTAT3 positive $^{\wedge}, n(\%)$ & S1PR2 positive, $n(\%)$ & $\begin{array}{l}\text { FOXP1 positive } *, n \\
(\%)\end{array}$ \\
\hline DLBCL, GCB & 43 & $0 / 43(0 \%)$ & $9 / 40(22 \%)$ & $0 / 43(0 \%)$ & $27 / 38(71 \%)$ \\
\hline DLBCL, non-GCB & 47 & $0 / 47(0 \%)$ & $23 / 45(51 \%)$ & $0 / 47(0 \%)$ & $39 / 46(85 \%)$ \\
\hline DLBCL, NA & 17 & $1 / 17(6 \%)$ & $6 / 17(35 \%)$ & $0 / 17(0 \%)$ & 16/17 (94\%) \\
\hline Total DLBCL & 107 & $1 / 107(1 \%)$ & $38 / 102(37 \%)$ & 0/107 (0\%) & $82 / 101(81 \%)$ \\
\hline $\begin{array}{l}\text { High grade B cell } \\
\text { lymphoma, double hit }\end{array}$ & 12 & $1 / 12(8 \%)$ & $3 / 12(25 \%)$ & $1 / 12(8 \%)$ & $12 / 12(100 \%)$ \\
\hline $\begin{array}{l}\text { High grade B cell } \\
\text { lymphoma, NOS }\end{array}$ & 15 & $2 / 15(13 \%)$ & $7 / 15(47 \%)$ & $4 / 15(27 \%)$ & $14 / 15(93 \%)$ \\
\hline Burkitt lymphoma & 15 & $0 / 15(0 \%)$ & $0 / 15(0 \%)$ & $0 / 15(0 \%)$ & $15 / 15(100 \%)$ \\
\hline $\begin{array}{l}\text { Burkitt-like lymphoma, Del } \\
11 \mathrm{q}\end{array}$ & 1 & $0 / 1(0 \%)$ & $1 / 1(100 \%)$ & $1 / 1(100 \%)$ & $1 / 1(100 \%)$ \\
\hline Total number of cases & 150 & 4 & 49 & 6 & 124 \\
\hline
\end{tabular}

FOXP1+ DLBCL, GCB (27/38 cases, 71\%) vs. FOXP1+ HGBL group $(26 / 27,96 \%)$ (Fisher's exact test, two-tailed $P$ value $=0.0103)$. FOXP1+ DLBCL, non-GCB type (39/46 cases, $85 \%)$ vs. FOXP1+ DLBCL, GCB (27/38 cases, $71 \%)$ (Fisher's exact test, two-tailed $P$ value $=0.1818)$, and FOXP1+ DLBCL, non-GCB type (39/46 cases, $85 \%)$ vs. FOXP1+ HGBL $(26 / 27,96 \%)$ (Fisher's exact test, two-tailed $P$ value $=0.2441)$

pSTAT3+ DLBCL, non-GCB (23/45 cases, 51\%), vs. pSTAT3+ DLBCL, GCB-type group $(9 / 40$ cases, $22 \%)($ Fisher's exact test, two-tailed $P$ value $=$ $0.0078)$ and pSTAT3+ DLBCL, non-GCB (23/45 cases, 51\%) vs. HGBL group (10/27 cases, 37\%) (Fisher's exact test, two-tailed $P$ value $=0.3296)$. MYC-driven lymphomas (HGBL, DH and BL) vs. HGBL, NOS revealed a statistically significant difference only for pSTAT3: (3/27, 11\%) vs (7/15, $47 \%$ ) (Fisher's exact test, two-tailed $P$ value $=0.0202)$ and borderline significance for S1PR2 $(1 / 27,4 \%)$ vs. $(4 / 15,27 \%)$ (Fisher's exact test, two-tailed $P$ value $=0.0469)$. MYC-driven lymphomas $(H G B L, D H$, and BL) vs. DLBCL, NOS revealed a statistically significant difference only for pSTAT3 $(3 / 27$, $11 \%)$ vs $(38 / 102,37 \%)$ (Fisher's exact test, two-tailed $P$ value $=0.0101)$ and FOXP1 $(27 / 27,100 \%)$ vs. $(82 / 101,81 \%)$ (Fisher's exact test, two-tailed $P$ value $=0.0125)$

*FOXP1+ cases: For BL: 14/15 showed staining in >90\% tumor cells; $1 / 15$ showed staining in $50-90 \%$ tumor cells. For HBL, NOS: $12 / 14$ showed staining in $>90 \%$ tumor cells; $2 / 14$ showed staining in 50-90\% tumor cells. For HBL, DH: 10/12 showed staining in > 90\% tumor cells; $2 / 12$ showed staining in 50-90\% tumor cells. For DLBCL, NA: 12/16 showed staining in $>90 \%$ tumor cells; 4/16 showed staining in 50-90\% tumor cells. For DLBCL, GCB-type: $24 / 27$ showed staining in >90\% tumor cells; $3 / 27$ showed staining in $50-90 \%$ tumor cells. For DLBCL, non-GCB-type: $33 / 39$ showed staining in $>90 \%$ tumor cells; $6 / 39$ showed staining in 50-90\% tumor cells. ${ }^{\wedge}$ Up to 5 DLBCL cases did not have remaining tissue available/ appropriate for scoring for FOXP1 and pSTAT3

43 cases of DLBCL, GCB type; 47 cases of DLBCL, nonGCB type; 17 cases of DLBCL, NA (i.e., subtyping not available); 12 cases of double-hit, high-grade B cell lymphoma (HGBL-DH); 15 cases of high-grade B cell lymphoma, NOS (HGBL, NOS); 15 cases of Burkitt lymphoma (BL); and 1 case of Burkitt-like lymphoma (BLL) with Del11q. The cases comprised the following proportions of nodal and extranodal tissues: BL: nodal: 3/15(20\%)/extranodal: 12/ 15(80\%); HGBL, DH: nodal: 4/12(33\%)/extranodal: 8/12(67\%); HGBL, NOS: nodal: 4/15(27\%)/extranodal: 11/ 15(73\%); DLBCL, GCB: nodal: 23/43 (53\%)/extranodal: 20/43(47\%); DLBCL, non-GCB: nodal: 26/47(55\%)/ extranodal: 21/47(45\%); DLBCL, NA: nodal: 9/17(53\%)/ extranodal: 8/17(47\%); BLL: extranodal: 1/1(100\%).

BL cases were negative for S1PR1, pSTAT3, and S1PR2, but were uniformly positive for FOXP1 (15/15 cases, 100\%). HGBL-DH were also uniformly positive for FOXP1 (12/12 cases, $100 \%$ ) and mostly negative for S1PR1, pSTAT3, and S1PR2, similar with Burkitt lymphoma; however, a small subset of the HGBCL-DH cases were positive for pSTAT3 (3/12 cases, 25\%), S1PR1 (1/12 cases, 8\%) and S1PR2 (1/12 cases, $8 \%)$. Interestingly, HGBL, NOS cases also showed near uniform expression of FOXP1 (14/15 cases, 93\%), but showed proportionally higher percentages of pSTAT3 (7/15 cases, 47\%), S1PR1 (2/15 cases, 13\%), and S1PR2 (4/15 cases, $27 \%$ ) positive cases.

DLBCL cases were analyzed according to GCB and nonBCB subtypes according to the Hans algorithm [1]. DLBCL, GCB-type cases showed a significantly lower proportion of FOXP1 positive cases (27/38 cases, $71 \%)$ than the HGBL group $(26 / 27,96 \%)$ (Fisher's exact test, two-tailed $P$ value $=$ 0.0103). The DLBCL, GCB-type cases also showed pSTAT3 positivity in $22 \%$ of cases (9/40 cases) and were uniformly negative for S1PR1 and S1PR2. The DLBCL, non-GCB type cases showed a slightly higher proportion of FOXP1 positive cases (39/46 cases, $85 \%$ ) compared to DLBCL, GCB-type cases, but this difference was not significant (Fisher's exact test, two-tailed $P$ value $=0.1818$ ). The proportion of FOXP1 positive DLBCL, non-GCB cases was also not significantly different from the HGBL group (Fisher's exact test, two-tailed $P$ value $=0.2441)$. The DLBCL, non-GCB type cases showed pSTAT3 positivity in $51 \%$ of cases ( $23 / 45$ cases), which was significantly higher than the DLBCL, GCB-type group (9/40 cases, 22\%) (Fisher's exact test, two-tailed $P$ value $=0.0078$ ), 


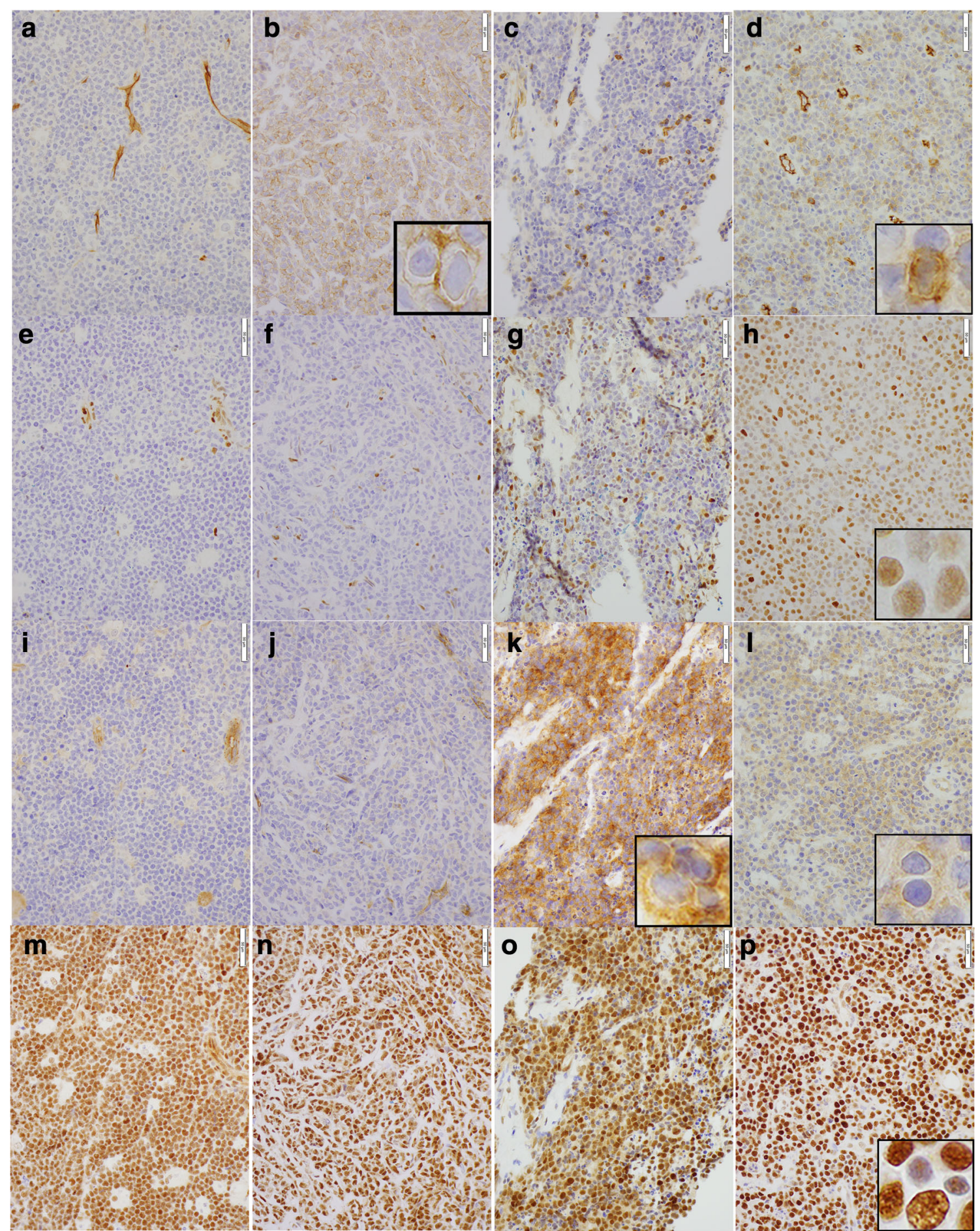

Fig. 2 Representative immunostaining for S1PR1 (a-d), pSTAT3 (e-h), S1PR2 (i-l), and FOXP1 (m-p) in four different cases: Burkitt lymphoma $(\mathbf{a}, \mathbf{e}, \mathbf{i}, \mathbf{m})$; HGBL-DH (b, f, j, n); HGBL, NOS (c, $\mathbf{g}, \mathbf{k}, \mathbf{o})$; and HGBL, $\operatorname{NOS}(\mathbf{d}, \mathbf{h}, \mathbf{l}, \mathbf{p})$. Burkitt lymphoma shows positivity for FOXP1. A HGBL-DH case $(\mathbf{b}, \mathbf{f}, \mathbf{j}, \mathbf{n})$ shows positivity for S1PR1 (b) and FOXP1

and was not significantly different than HGBL group (10/27 cases, 37\%) (Fisher's exact test, two-tailed $P$ value $=0.3296$ ). The GCB-type and non-GCB-type subgroups of DLBCL did not show significant levels of S1PR1 or S1PR2 expression. (n). A HGBL, NOS case (c, $\mathbf{g}, \mathbf{k}, \mathbf{0})$ shows positivity for S1PR2 (k) and FOXP1 (o). A separate HGBL, NOS case $(\mathbf{d}, \mathbf{h}, \mathbf{l}, \mathbf{p})$ shows variable positivity for S1PR1 (d), pSTAT3 (h), S1PR2 (l), and FOXP1 (p). Images are shown at $\times 40$ magnification. Scale bars $=50 \mu \mathrm{m}$

In terms of S1PR1 and S1PR2 expression, 6\% (9/150) of all cases were positive for S1PR1 and/or S1PR2: $2 \%$ (3/150) of cases were S1PR1+, 3\% (5/150) were S1PR2+, and 1 case $(1 / 150,<1 \%)$ was positive for both S1PR1 and S1PR2. Thus, 
S1PR1 and S1PR2 staining was mutually exclusive in $89 \%$ (8/9) of these cases. The features of the S1PR1+ and S1PR2+ cases are described in Table 2 . Such cases were predominantly HGBL. Due to the low prevalence of S1PR1+ and S1PR2+ cases, the respective relationship to pSTAT3 and FOXP1 staining was not possible to ascertain; the four S1PR1+ cases included both pSTAT3+ (2/4) and pSTAT3-negative cases (2/4). The six S1PR2+ cases were all FOXP1 positive (6/6).

Lastly, comparison of MYC-driven lymphomas (HGBL, DH and BL) vs. HGBL, NOS for the four immunohistochemical markers revealed a statistically significant difference only for pSTAT3 (Fisher's exact test, two-tailed $P$ value $=0.0202$ ) and a borderline significance for S1PR2 (Fisher's exact test, two-tailed $P$ value $=0.0469$ ). Additionally, comparison of MYC-driven lymphomas (HGBL, DH, and BL) vs. DLBCL, NOS for the four immunohistochemical markers revealed a statistically significant difference only for pSTAT3 (Fisher's exact test, two-tailed $P$ value $=0.0101$ ) and FOXP1 (Fisher's exact test, two-tailed $P$ value $=0.0125$ ) .

\section{Discussion}

Our study examined the expression of S1PR1, pSTAT3, S1PR2, and FOXP1 using immunostaining of formalin-fixed paraffin embedded patient samples from a clinical cohort of a variety of aggressive B cell lymphoma cases. This was done in an attempt to extend our understanding of these markers beyond DLBCL, given that these potentially important pathways have not been adequately studied in BL and HGBL$\mathrm{DH}$, etc.

In prior studies of DLBCL, Koresawa et al. [5] reported $13 \%$ of their DLBCL cases were S1PR1 positive and were enriched for DLBCL, non-GCB type; interestingly, they found that primary testicular DLBCL cases showed a higher prevalence of S1PR1 positivity (54\%). Thus, the overall prevalence of S1PR1+ cases in their cohort was approximately $10 \%$, when considering only DLBCL cases which were not primary testicular DLBCL. They found that among early stage of DLBCL patients, S1PR1 expression was associated with a poor prognosis. Paik et al. [16] reported S1PR1 positivity in $40 \%$ of their DLBCL cases and the S1PR1+ cases also appeared enriched for DLBCL occurring in extranodal sites, but there was no significant difference for S1PR expression between the GCB-type and non-GCB-type subgroups; Paik et al. also found that S1PR1 expression was associated with a poor prognosis. Lastly, Nishimura et al. [4] reported that $6 \%$ of DLBCL cases were S1PR1 positive. The differences in the prevalence of S1PR1 positivity among the different published cohorts may be due to several factors. In our study, we have found that approximately $1 \%(1 / 107)$ of DLBCLs were positive for S1PR1 and we found that S1PR1 was expressed in approximately $11 \%$ (3/27) of HGBLs. Overall, the prevalence of S1PR1+ DLBCL and S1PR1+ HGBL in our study are in a similar range as the prevalence of S1PR1 positivity reported by Koresawa et al. [5] and Nishimura et al. [4] (i.e., 6-10\%). Although, the prevalence of S1PR1+ DLBCL in our study was slightly less than that seen by Koresawa et al. [5] and Nishimura et al. [4], the collective findings from these studies point to a consensus that S1PR1+ cases represent a small subset of DLBCL (excluding primary testicular DLBCL). Furthermore, the prior publications are somewhat limited in the sense that they do not clearly indicate the specific proportion, if any, of HGBL in their cohorts, which may also contribute to the slight differences in prevalence, since we observed a higher prevalence of S1PR1 positivity in HGBL than in DLBCL, which was statistically significant (Fisher's exact test, two-tailed $P$ value $=0.0257$ ). In terms of primary testicular DLBCL, our cohort included only 2 cases of primary testicular DLBCL which were both negative for S1PR1. In addition to the above considerations regarding differences in cohorts, although some of the prior studies, like our study, use FFPE and the same primary anti-S1PR1 antibody, technical differences (e.g., variability in the formalin fixation, automated staining platforms and/or variability in the cytoplasmic/ membrane staining patterns reported by others $[5,16]$ ) may contribute to variability in S1PR1 prevalence between the studies. Lastly, the cutoff for S1PR1 positivity in the

Table 2 Details of S1PR1+ and S1PR2+ cases

\begin{tabular}{lllllll}
\hline Case \# & Diagnosis & S1PR IHC & pSTAT3 IHC & FOXP1 IHC & Cytogenetic rearrangements & Anatomic location \\
\hline 55 & DLBCL, NA & S1PR1+/S1PR2- & Negative & Positive & NA & Brain \\
4 & HGBL-DH & S1PR1-/S1PR2+ & Positive & Positive & MYC+, BCL6+, BCL2- & Pelvic mass \\
8 & HGBL-NOS & S1PR1-/S1PR2+ & Positive & Positive & MYC-, BCL6-, BCL2- & Thyroid mass \\
9 & HGBL-NOS & S1PR1-/S1PR2+ & Positive & Positive & MYC-, BCL6-, BCL2- & Brain \\
10 & HGBL-NOS & S1PR1+/S1PR2+ & Positive & Positive & MYC-, BCL6-, BCL2- & Brain \\
20 & BLL-Del(11)(q23;q25) & S1PR1-/S1PR2+ & Positive & Positive & MYC-, BCL6-, BCL2- & Pharyngeal wall \\
26 & HGBL-NOS & S1PR1-/S1PR2+ & Negative & Positive & MYC-, BCL6-NA, BCL2-NA & Vulva \\
38 & HGBL-DH & S1PR1+/S1PR2- & Negative & Positive & MYC+, BCL6-, BCL2+ & Lymph node \\
51 & HGBL-NOS & S1PR1+/S1PR2- & Positive & Positive & MYC-, BCL6-, BCL2-NA & Nasal mass \\
\hline
\end{tabular}


Koresawa et al. [5] and Paik et al. [16] papers was only 20$30 \%$ of tumor cells, which would include cases with S1PR1 staining in only a small subset of tumor cells that may not be representative of the overall tumor, whereas we applied a more robust/stringent threshold of $\geq 50 \%$ tumor cells; this may also contribute to the slightly higher S1PR1 prevalence in prior studies.

In previous studies of pSTAT3 in DLBCL [16, 26, 27], phospho-STAT3 (Tyr705) staining was found in $32-37 \%$ of DLBCL cases and appeared enriched in DLBCL, non-GCBtype; one of these studies reported an even higher prevalence (59\%) of pSTAT3 positivity in DLBCL [16]. High nuclear expression of STAT3 and phosphoSTAT3 have been associated with unfavorable prognosis in DLBCL subgroups in these studies. Interestingly, S1PR1 has been reported to be transcribed by pSTAT3 and S1PR1 reportedly can, in turn, activate STAT3 in a positive feedback loop [15]. Furthermore, S1PR1 has been reported to be an effective target to block STAT3 signaling, tumor cell growth and metastatic spread using primary lymphoma cell models and DLBCL cell lines in vitro and in vivo [17]; however, the potential relationship between S1PR1 and pSTAT3 has not been adequately studied in clinical material from patients with DLBCL and other aggressive B cell lymphomas. In our cohort, we found that $38 \%$ of DLBCL cases (38/102) were positive for pSTAT3. This is in line with several prior studies mentioned above (32-37\%). In addition, we found that HGBL also showed a similar prevalence of pSTAT3+ cases $(37 \%, 10 / 27)$. Our study agrees with prior studies where pSTAT3 was expressed more frequently in non-GCB-type, DLBCL cases compared to GCB-type, DLBCL cases [16, 17, 26, 27]. Lastly, our BL cases appeared to be uniformly negative for pSTAT3, and therefore, appear different from DLBCL and HGBL. Indeed, when we compared MYC-driven lymphomas (BL and $\mathrm{HGBL}, \mathrm{DH}$ ) as a group with HGBL, NOS and DLBCL, there was a significantly lower prevalence of pSTAT3 positivity in the MYC-driven lymphomas compared to both HGBL, NOS and DLBCL. Given the low number of S1PR1+ cases in our study, any potential relationship between S1PR1 and pSTAT3 expression could not be assessed in our cohort. Importantly, Koresawa et al. [5] did not observe a relationship between S1PR1 positivity and STAT3 phosphorylation among DLBCL cases by IHC analysis of FFPE sections. However, in a subset of their cases with frozen tissue available, Koresawa et al. [5] did observe a correlation between S1PR1 expression and phosphorylation of STAT3 by Western blot analysis. Similarly, Liu et al. [17] also observed a correlation between S1PR1 expression and phospho-STAT3 using primary tumor cells from 10 non-GCB-type, DLBCL patient samples, and 2 DLBCL cell lines. These reported findings underscore the known difficulty of immunostaining of FFPE for phospho-epitope markers such as p-STAT3 due to tissue fixation and tissue processing factors [5] and should be taken into consideration for future studies that explore the potential relationship between S1PR1 and pSTAT3 expression from clinical material.

FOXP1 is a transcription factor that typically functions as a transcriptional repressor and tends to be more highly expressed in DLBCL, non-GCB-type (up to $71 \%$ of cases) compared to GCB-type cases [20,22]. Moderate to high expression of FOXP1 in a high percentage of tumor cells has been associated with DLBCL, non-GCB-type and a trend toward inferior outcome in DLBCL [20]. In terms of the S1PR2/ FOXP1 axis, Flori et al. [22] examined FOXP1 expression and the relationship to S1PR2 expression in cell lines of both GCB-type and Non-GCB-type DLBCL. They found that strong expression of FOXP1 was more commonly observed in Non-GCB-type cell lines compared to GCB-type cell lines. Through FOXP1 siRNA knockdown in cell lines, RNA sequencing and FOXP1-specific antibody chromatin immunoprecipitation, Flori et al. identified S1PR2 as pro-apoptotic factor (i.e., a tumor suppressor) both in vitro and in vivo, which is directly repressed by FOXP1. They also noted that the expression of S1PR2 was inversely proportional to FOXP1 expression in publicly available gene expression profiling studies from patient samples. Importantly, they found that high FOXP1 and low S1PR2 transcript levels were associated with inferior survival. The tumor suppressive properties of S1PR2 had also been previously reported by Muppidi et al. [24] who found that in mouse models, heterozygous loss of S1 pr2 led to marked expansion of germinal centers, that could be repressed by overexpression of wild type S1PR2, but not a mutant S1PR2 incapable of signaling thru Gna13 and Arhgef1 to regulate cell growth and migration. They also found evidence for the importance of the S1PR2/GNA13/ARHGEF1 pathway in human DLBCL cell lines. This was consistent with earlier mouse model work by Green et al. [23] which also revealed an important role of S1pr2 in germinal center B cell homeostasis, whereby S1pr2 expression in germinal center B cells regulated apoptosis of germinal center B cells and also regulated germinal center B cell confinement via Gna12/Gna13/Arhgef1. Lastly, early studies by Cattoretti et al. [6] also found evidence that S1pr2 could act as a tumor suppressor in mouse models where S1pr2-deficient mice showed a tendency to develop lymphoma (GCB-type DLBCL) with aging. In terms of FOXP1 in our study, we have found that $\mathrm{BL}$ cases showed a unique pattern of expression with strong expression on FOXP1 in all cases, whereas these BL cases lacked expression of S1PR1, pSTAT3, and S1PR2. The absence of pSTAT3 staining in Burkitt lymphoma (a tumor of postulated germinal center B cell origin) appears consistent with the previously reported association of pSTAT3 expression in non-GCB type, DLBCL. Also, the inverse expression pattern of FOXP1 and S1PR2 would appear to be consistent with the findings by Flori et al. [22] whereby expression of FOXP1 and S1PR2 were inversely proportional. The uniform expression of FOXP1 in our BL cases is 
somewhat interesting given that FOXP1 has been reported to be highly expressed in the context of non-GCB type, DLBCL (although expression in GCB-type DLBCL has been described). Among our HGBL cases, we found that FOXP1 was expressed in 96\% (26/27) of cases and in our DLBCL cases, we found that FOXP1 was expressed in $81 \%(82 / 101)$ and was not significantly different between GCB-type and non-GCB-type DLBCL subgroups. The high prevalence of FOXP1+ HGBL cases (which is slightly higher than the prevalence of FOXP1+ DLBCL cases and approaches the prevalence of FOXP1+ BL cases in our cohort), is compatible with the concept that HGBL cases often demonstrate "Burkitt-like" cytogenetic (i.e., MYC translocation), morphologic, and immunohistochemical features, which are intermediate between DLBCL and BL.

Lastly, S1PR2 was expressed in approximately 19\% (5/27) of HGBLs and in the case of Burkitt-like lymphoma with Del11q, but was negative in DLBCLs and BLs. The detectable expression of S1PR2 may at first appear counterintuitive, given that it is a putative tumor suppressor gene; however, loss of function mutations in S1PR2 have been described in DLBCL [24], and for other biomarkers (e.g., TP53), a precedent for detectable immunostaining in the presence of inactivating mutations has been described $[28,29]$. Genotyping data of S1PR1 and S1PR2 are not available in our cases. Interestingly, in some of our case material, it was noted that S1PR2 was most strongly expressed in areas with high levels of apoptosis (e.g., Fig. 1: germinal center; Fig. 2, panel K). Overall, among cases positive for S1PR staining, S1PR1 and S1PR2 staining were mutually exclusive in most cases $(89 \%$, $8 / 9$ ), which is compatible with the prior concept that these receptors having antagonistic effects on cell growth and migration in several different cell systems [30]. As a category, HGBL comprised the majority of cases positive for S1PR1 or S1PR2 (78\%, 7/9 cases). Given the low number of S1PR+ cases in our cohort, there was no clear relationship with MYC, BCL6, or BCL2 translocation status or with immunostaining for pSTAT3 or FOXP1. Eighty-nine percent (8/9) of S1PR+ cases were extranodal lymphomas, compatible with the reported findings in DLBCL where S1PR1 expression was associated with extranodal lymphomas $[5,16]$.

One of the main limitations of our study is the small size of the cohort which, given the low prevalence of S1PR1 and S1PR2 immunostaining, limits the interpretation of the findings. Also, the limited availability of cytogenetic data in a subset of the DLBCL cases is another limitation of the study. Additional studies are required on larger cohorts of these lymphoma types, in order to more fully explore the potential relationship between S1PR1, pSTAT3, S1PR2 and FOXP1 in clinical samples. In addition, studies with genotyping data available may also be helpful to clarify any potential relationships between mutation status (i.e., genotype) and immunostaining (i.e., phenotype) of S1PR1 and/or S1PR2.
In conclusion, herein, we report the staining patterns of S1PR1, pSTAT3, S1PR2, and FOXP1 in a cohort of aggressive, mature B cell lymphomas. We have confirmed the previously reported expression of S1PR1 in mantle zones in reactive follicles and we have found that (i) S1PR2 showed expression in follicle centers in reactive lymphoid tissue, (ii) Burkitt lymphomas showed a unique pattern of expression compared to HGBL and DLBCL, (iii) S1PR1 and S1PR2 were expressed in a low proportion of cases, which were frequently HGBL involving extranodal sites and S1PR1, S1PR2 staining was predominantly mutually exclusive, (iv) FOXP1 was expressed in a high proportion of the various case types and, (v) pSTAT3, which represents a possible therapeutically targetable pathway, was detected in a significant proportion of HGBL and DLBCL cases. Taken together, these findings provide further evidence that S1PR1, pSTAT3, S1PR2, and FOXP1 play a role in a subset of aggressive mature B cell lymphomas and deserve future study.

Funding This work was supported by the (i) Department of Pathology and Laboratory Medicine, Weill Cornell Medicine (MJK and TS), (ii) American Heart Association (Grant-in-Aid, 12GRNT12050110) (TS), and (iii) NIH (HL094465) (TS). The TS laboratory is also supported by a grant from the Leducq Foundation.

\section{Compliance with ethical standards}

Conflict of interest MAK, TS, and MJK declare that they have no conflict of interest.

\section{References}

1. Swerdlow SH, Campo E, Harris NL et al (eds) (2008) WHO classification of tumours of haematopoietic and lymphoid tissues. 4th edition. International Agency for Research on Cancer, Lyon

2. Swerdlow SH, Campo E, Harris NL et al (eds) (2017) WHO classification of tumours of haematopoietic and lymphoid tissues. Revised 4th edition. International Agency for Research on Cancer, Lyon

3. Wasik AM, Wu C, Mansouri L, Rosenquist R, Pan-Hammarstrom Q, Sander B (2018) Clinical and functional impact of recurrent S1PR1 mutations in mantle cell lymphoma. Blood Adv 2:621-625

4. Nishimura H, Akiyama T, Monobe Y, Matsubara K, Igarashi Y, Abe M, Sugihara T, Sadahira Y (2010) Expression of sphingosine-1-phosphate receptor 1 in mantle cell lymphoma. Mod Pathol 23:439-449

5. Koresawa R, Yamazaki K, Oka D, Fujiwara H, Nishimura H, Akiyama T, Hamasaki S, Wada H, Sugihara T, Sadahira Y (2016) Sphingosine-1-phosphate receptor 1 as a prognostic biomarker and therapeutic target for patients with primary testicular diffuse large B-cell lymphoma. Br J Haematol 174:264-274

6. Cattoretti G, Mandelbaum J, Lee N, Chaves AH, Mahler AM, Chadburn A, Dalla-Favera R, Pasqualucci L, MacLennan AJ (2009) Targeted disruption of the S1P2 sphingosine 1-phosphate receptor gene leads to diffuse large B-cell lymphoma formation. Cancer Res 69:8686-8692

7. Li G, So AY, Sookram R et al (2018) Epigenetic silencing of miR$125 \mathrm{~b}$ is required for normal B-cell development. Blood 131:1920 1930 
8. Kluk MJ, Ryan KP, Wang B, Zhang G, Rodig SJ, Sanchez T (2013) Sphingosine-1-phosphate receptor 1 in classical Hodgkin lymphoma: assessment of expression and role in cell migration. Lab Investig 93:462-471

9. Vrzalikova K, Ibrahim M, Vockerodt M, Perry T, Margielewska S, Lupino L, Nagy E, Soilleux E, Liebelt D, Hollows R, Last A, Reynolds G, Abdullah M, Curley H, Care M, Krappmann D, Tooze R, Allegood J, Spiegel S, Wei W, Woodman CBJ, Murray PG (2018) S1PR1 drives a feedforward signalling loop to regulate BATF3 and the transcriptional programme of Hodgkin lymphoma cells. Leukemia 32:214-223

10. Middle S, Coupland SE, Taktak A, Kidgell V, Slupsky JR, Pettitt AR, Till KJ (2015) Immunohistochemical analysis indicates that the anatomical location of B-cell non-Hodgkin's lymphoma is determined by differentially expressed chemokine receptors, sphingosine-1-phosphate receptors and integrins. Exp Hematol Oncol 4:10

11. Kuusanmaki H, Dufva O, Parri E et al (2017) Drug sensitivity profiling identifies potential therapies for lymphoproliferative disorders with overactive JAK/STAT3 signaling. Oncotarget 8:9751697527

12. Lam LT, Wright G, Davis RE, Lenz G, Farinha P, Dang L, Chan JW, Rosenwald A, Gascoyne RD, Staudt LM (2008) Cooperative signaling through the signal transducer and activator of transcription 3 and nuclear factor- $\{$ kappa $\}$ B pathways in subtypes of diffuse large B-cell lymphoma. Blood 111:3701-3713

13. Petrakis G, Kostopoulos I, Venizelos I, Lambropoulou M, Vouras K, Vakalopoulou S, Mandala E, Tsatalas C, Papadopoulos N (2017) Expression of the activation markers Blimp1, Foxp1 and pStat3 in extranodal diffuse large B-cell lymphomas. Histol Histopathol 32: 825-834

14. Ding BB, Yu JJ, Yu RY, Mendez LM, Shaknovich R, Zhang Y, Cattoretti G, Ye BH (2008) Constitutively activated STAT3 promotes cell proliferation and survival in the activated B-cell subtype of diffuse large B-cell lymphomas. Blood 111:1515-1523

15. Lee H, Deng J, Kujawski M, Yang C, Liu Y, Herrmann A, Kortylewski M, Horne D, Somlo G, Forman S, Jove R, Yu H (2010) STAT3-induced S1PR1 expression is crucial for persistent STAT3 activation in tumors. Nat Med 16:1421-1428

16. Paik JH, Nam SJ, Kim TM, Heo DS, Kim CW, Jeon YK (2014) Overexpression of sphingosine-1-phosphate receptor 1 and phospho-signal transducer and activator of transcription 3 is associated with poor prognosis in rituximab-treated diffuse large B-cell lymphomas. BMC Cancer 14:911

17. Liu Y, Deng J, Wang L, Lee H, Armstrong B, Scuto A, Kowolik C, Weiss LM, Forman S, Yu H (2012) S1PR1 is an effective target to block STAT3 signaling in activated B cell-like diffuse large B-cell lymphoma. Blood 120:1458-1465

18. Zeng R, Tang Y, Zhou H, Liu Y, Huang J, Li L, Liu W, Feng Y, Zhou Y, Chen T, Zhang L, Zhong M (2017) STAT3 mediates multidrug resistance of Burkitt lymphoma cells by promoting antioxidant feedback. Biochem Biophys Res Commun 488:182-188

19. Ok CY, Chen J, Xu-Monette ZY, Tzankov A, Manyam GC, Li L, Visco C, Montes-Moreno S, Dybkaer K, Chiu A, Orazi A, Zu Y, Bhagat G, Richards KL, Hsi ED, Choi WWL, van Krieken JH, Huh J, Zhao X, Ponzoni M, Ferreri AJM, Bertoni F, Farnen JP, Moller MB, Piris MA, Winter JN, Medeiros LJ, Young KH (2014) Clinical implications of phosphorylated STAT3 expression in De novo diffuse large B-cell lymphoma. Clin Cancer Res 20:5113-5123

20. Gascoyne DM, Banham AH (2017) The significance of FOXP1 in diffuse large B-cell lymphoma. Leuk Lymphoma 58:1037-1051

21. Wang W, Hu S, Lu X, Young KH, Medeiros LJ (2015) Triple-hit Bcell lymphoma with MYC, BCL2, and BCL6 translocations/rearrangements: clinicopathologic features of 11 cases. Am J Surg Pathol 39:1132-1139

22. Flori M, Schmid CA, Sumrall ET, Tzankov A, Law CW, Robinson MD, Muller A (2016) The hematopoietic oncoprotein FOXP1 promotes tumor cell survival in diffuse large B-cell lymphoma by repressing S1PR2 signaling. Blood 127:1438-1448

23. Green JA, Suzuki K, Cho B, Willison LD, Palmer D, Allen CDC, Schmidt TH, Xu Y, Proia RL, Coughlin SR, Cyster JG (2011) The sphingosine 1-phosphate receptor $\mathrm{S} 1 \mathrm{P}(2)$ maintains the homeostasis of germinal center B cells and promotes niche confinement. Nat Immunol 12:672-680

24. Muppidi JR, Schmitz R, Green JA, Xiao W, Larsen AB, Braun SE, An J, Xu Y, Rosenwald A, Ott G, Gascoyne RD, Rimsza LM, Campo E, Jaffe ES, Delabie J, Smeland EB, Braziel RM, Tubbs RR, Cook JR, Weisenburger DD, Chan WC, Vaidehi N, Staudt LM, Cyster JG (2014) Loss of signalling via Galpha13 in germinal centre B-cell-derived lymphoma. Nature 516:254-258

25. Morin RD, Mungall K, Pleasance E, Mungall AJ, Goya R, Huff RD, Scott DW, Ding J, Roth A, Chiu R, Corbett RD, Chan FC, Mendez-Lago M, Trinh DL, Bolger-Munro M, Taylor G, Hadj Khodabakhshi A, Ben-Neriah S, Pon J, Meissner B, Woolcock B, Farnoud N, Rogic S, Lim EL, Johnson NA, Shah S, Jones S, Steidl C, Holt R, Birol I, Moore R, Connors JM, Gascoyne RD, Marra MA (2013) Mutational and structural analysis of diffuse large Bcell lymphoma using whole-genome sequencing. Blood 122:12561265

26. Huang X, Meng B, Iqbal J, Ding BB, Perry AM, Cao W, Smith LM, Bi C, Jiang C, Greiner TC, Weisenburger DD, Rimsza L, Rosenwald A, Ott G, Delabie J, Campo E, Braziel RM, Gascoyne RD, Cook JR, Tubbs RR, Jaffe ES, Armitage JO, Vose JM, Staudt LM, McKeithan TW, Chan WC, Ye BH, Fu K (2013) Activation of the STAT3 signaling pathway is associated with poor survival in diffuse large B-cell lymphoma treated with R-CHOP. J Clin Oncol 31:4520-4528

27. Wu ZL, Song YQ, Shi Y, Zhu J (2011) High nuclear expression of STAT3 is associated with unfavorable prognosis in diffuse large Bcell lymphoma. J Hematol Oncol 4:31

28. Yemelyanova A, Vang R, Kshirsagar M, Lu D, Marks MA, Shih IM, Kurman RJ (2011) Immunohistochemical staining patterns of p53 can serve as a surrogate marker for TP53 mutations in ovarian carcinoma: an immunohistochemical and nucleotide sequencing analysis. Mod Pathol 24:1248-1253

29. Kazantseva M, Hung NA, Mehta S, Roth I, Eiholzer R, Rich AM, Seo B, Baird MA, Braithwaite AW, Slatter TL (2017) Tumor protein 53 mutations are enriched in diffuse large B-cell lymphoma with irregular CD19 marker expression. Sci Rep 7:1566

30. Sanchez T (2016) Sphingosine-1-phosphate signaling in endothelial disorders. Curr Atheroscler Rep 18:31

Publisher's note Springer Nature remains neutral with regard to jurisdictional claims in published maps and institutional affiliations. 